\title{
Exploration: Past and Future Contributions of the Vertical Lift Community and the Flight Vehicle Research and Technology Division
}

\author{
Larry A. Young $^{*}$ and Edwin W. Aiken ${ }^{\dagger}$ \\ NASA Ames Research Center, Moffett Field, CA 94035
}

\begin{abstract}
Fulfillment of the exploration vision will require new cross-mission directorate and multi-technical discipline synergies in order to achieve the necessary long-term sustainability. In part, lessons from the Apollo-era, as well as more recent research efforts, suggest that the aeronautics - and specifically the vertical lift - research community can and will make significant contributions to the exploration effort. A number of notional concepts and associated technologies for such contributions are outlined.
\end{abstract}

\section{Introduction}

$\mathrm{D}$ URING the Apollo-era, the vertical lift research community made noteworthy contributions to NASA's reaching the moon, in an addition to its more conventional ongoing research into rotorcraft and vertical take-off and landing aircraft for terrestrial applications. For the new exploration vision, the vertical lift community can undoubtedly contribute yet again. The Flight Vehicle Research and Technology Division (formerly known as the "Army/NASA Rotorcraft Division") has been active for several years in attempting to make, in a modest way, technical contributions from traditional aeronautics (specifically aeromechanics, flight dynamics and control) to NASA planetary science and exploration goals.

It is often the norm in today's world of engineering to engage in overspecialization. This, in itself, in a relatively stable technological environment, is not necessarily a bad thing. However, under circumstances of rapid change and transformation, this is a potentially fatal flaw. In particular, a narrowly focused "stove-piped" technical approach fails to allow for the necessary pursuit of truly crosscutting technologies and innovations. Only through broadening the spectrum of technologies and disciplines that can potentially contribute to NASA's transformation and focus on the exploration vision, can true sustainability be achieved.

This paper discusses some of the historical precedents for aeronautics and astronautics cross-pollination as well as summarizes some current pertinent research efforts and possible future opportunities. In a small way, these early efforts on the part of the NASA Ames Flight Vehicle Research and Technology Division perhaps represent a model of how core competencies and transformation can be satisfactorily balanced in this new age of exploration ${ }^{1,5}$.

\section{Apollo-Era Contributions of VTOL}

Among the most recognizable examples of contributions of vertical takeoff and landing technology to the Apollo program was the precursor technology development and training efforts related to, and in support of, the lunar excursion module (LEM). In addition to fixed- and moving-base simulators being developed, the LLRF (lunar landing research facility) and the LLRV (lunar landing research vehicle) were developed and tested at NASA Langley and Dryden. In many ways these flight test and training facilities drew heavily upon vertical takeoff and landing aircraft concepts and technologies. Figures 1-2 are NASA archive photographs of the LLRV and LLRF.

\footnotetext{
* Aerospace Engineer, Flight Vehicle Research and Technology Division, Mail Stop 243-12, Senior Member.

${ }^{\dagger}$ Division Chief (retired), Army/NASA Rotorcraft Division, Mail Stop 243-10.
} 


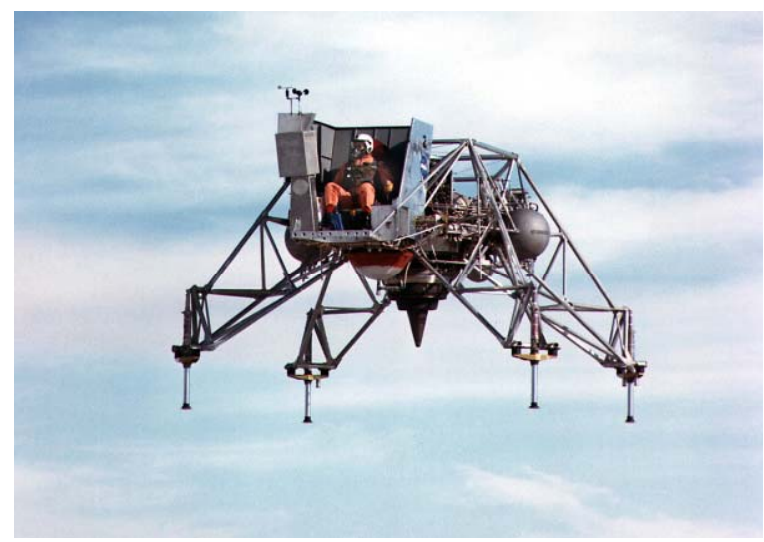

Figure 1. LLRV Flight Testing (NASA Photo \# ECN-1606)

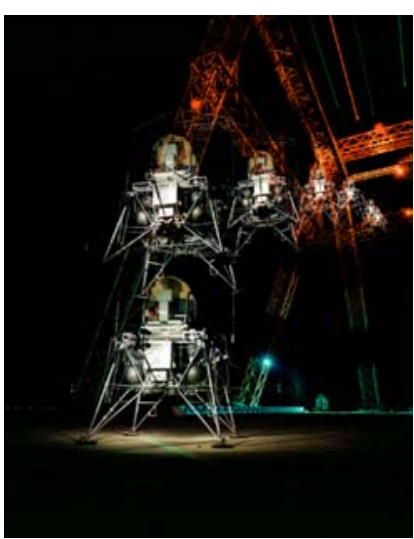

Figure 2. LLRF Operation (NASA \# GPN-2000-001281)

In addition to LLRV and LLRF facility development and test operations, there was also a balanced program of small- and large-scale prototype development, leading up to the LEM development (refer, for example, to the NASA Langley archive photographs in Fig. 3a-b). Even wind tunnel facilities were used to measure and assess the flight handling qualities of these surrogate lander flight platforms.
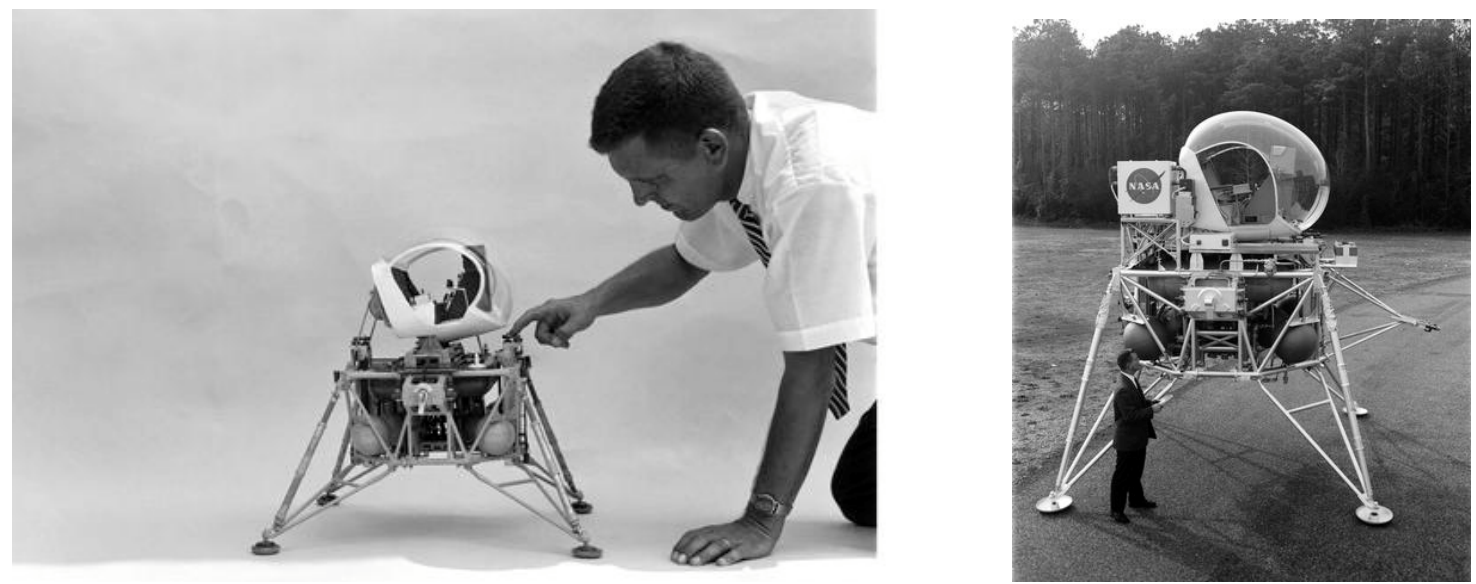

Figure 3a-b. Importance of Testing at Multiple Scales with Surrogate Platforms (NASA \# EL-2002-00380 and EL2000-00431)

It is key contention of this paper that similar synergistic aeronautics and astronautics research and development efforts such as those that were undertaken for the Apollo program will be required to develop Crew Exploration Vehicle (CEV), lander, and planetary surface operation system of systems elements for Project Constellation.

\section{More Recent, but Pre-Vision, Efforts}

The Flight Vehicle Research and Technology Division (in its current form and in its earlier incarnation as the Army/NASA Rotorcraft Division) has progressively over the past seven years been making modest inroads into the development of innovative technologies and concepts for planetary science and exploration applications. Until recently, most of the work has focused upon developing aerial vehicle concepts and autonomous system technologies for robotically exploring other planetary bodies, such as Mars, Venus, Titan, and the outer gas giant planets. In addition to performing and reporting several conceptual design studies ${ }^{6-9}$, for example, the Division has 
sponsored experimental and computational fluid dynamics analyses for rotors and VTOL vehicles operating in the unique operating environments of these planetary bodies - particularly Mars. The Division also has cosponsored/supported university-level student design competitions on this topic area ${ }^{16-18}$.

With the renewed emphasis from the exploration vision of a return to the moon as a steppingstone and proving ground for technologies for Mars and beyond, the Division has been examining leveraging previous exploration-related work to the goals and objectives of the vision. Therefore, for example, "robotic symbiote" concepts principally developed for aerial explorers ${ }^{3,4,8}$ are being revisited for possible lunar campaigns, using ballistic rather than aerial vehicle deployment, under the BADDEST (Ballistic Assisted Delivery of Devices to Efficiently Study Terrain) concept. Alternatively, bio-inspired autonomy concepts developed for flight control and search and find strategies for aerial explorers can be adapted for the autonomy component of the "palanquin" EVA/rover surface environmental management system problem. Classic man-in-the-machine human cognition/behavioral modeling used for rotorcraft/system human factors problem-solving can be extended into a new application domain to develop a series of teleoperated and/or semi-autonomous "universal anthropomorphic processors (UAP)" for enhanced risk mitigation "test driving" and flight certifying human-rated equipment for future exploration campaigns.

\section{Achieving a Satisfactory Balance}

Figure 4 shows schematically how various exploration concepts/technologies have been progressively developed, and in many instances, leveraged off of previous work, within the Division. All of this work, though, stems from a firm grounding in aeronautics research competencies. Such lineage - and a satisfactory balance between aeronautics and astronautics work - is essential to preserve these competencies while at the same time promoting innovation into new application domains. Each of these exploration system concepts will be discussed later in the paper.

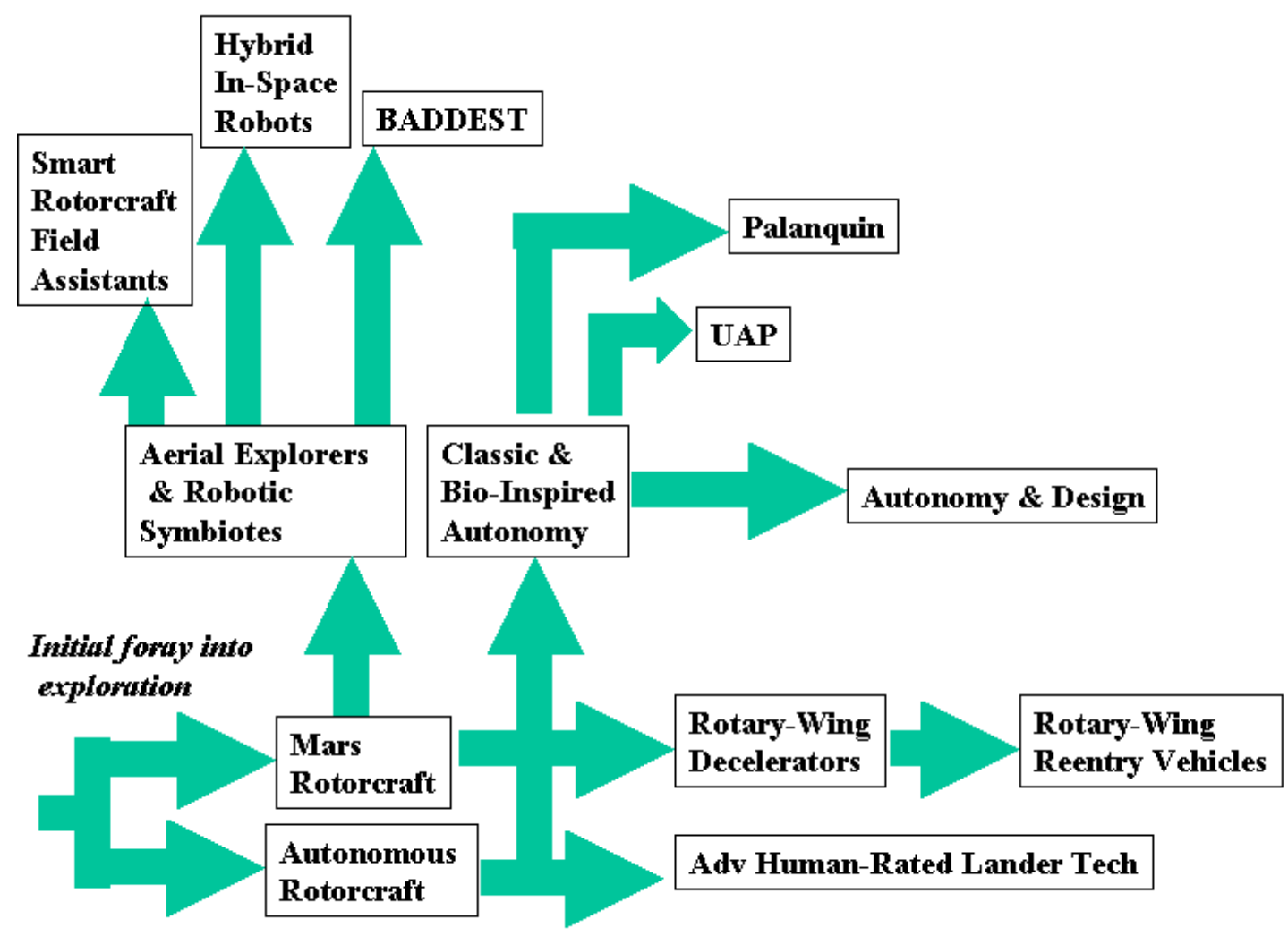

Figure 4. Progressively Defining New Technologies and Exploration Options 


\section{Future Potential Contributions}

Common themes inherent in the exploration-related research of the Division include: 1. the search for aeronautical (principally aerodynamics, flight controls, and human factor) technology dual-use application to exploration needs, 2. development/sponsorship of autonomous system technology of various forms as applied to advanced/adaptive systems and real-time mission-critical applications, and 3. fostering the infusion of robotics technology into both aeronautic and astronautic applications to yield wholly new capabilities. These research themes have manifested themselves in several specific technology/concept efforts within the Division and collaboration with other (in particular science and information technology) organizational partners.

\section{A. Aerial Explorers (Fixed-Wing and VTOL)}

The Flight Vehicle Research and Technology Division has conducted a considerable amount of work to date on vertical lift planetary aerial vehicles. The key emphasis of this work has been on establishing the feasibility of small robotic rotorcraft that can fly through the lower (near-surface) atmosphere of Mars (Fig. 5). The Mars rotorcraft project is also an excellent example of how the vertical lift research community, as a whole, can and has contributed to the exploration vision; an AHS student design competition was held in 2000 wherein several promising Mars rotorcraft concepts and technologies were identified ${ }^{16-17}$. A parallel line of investigation with respect to the Mars rotorcraft effort is the study of smart rotorcraft field assistants (SRFA) ${ }^{6}$ for field science investigations in terrestrial extreme environments (Fig. 6).

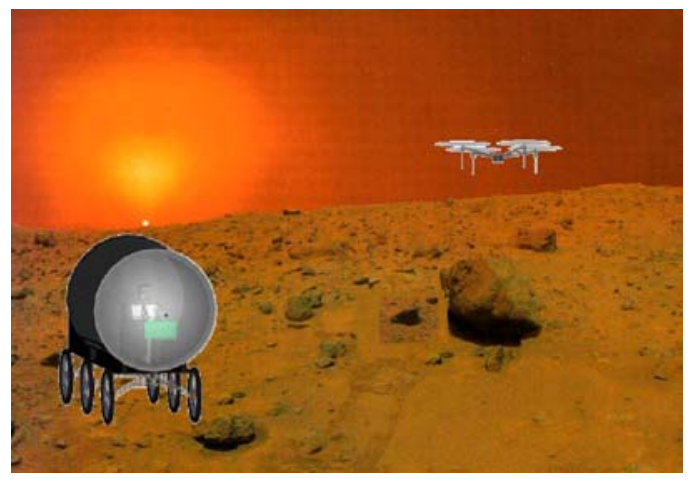

Figure 5. Mars Rotorcraft as Astronaut Assistants

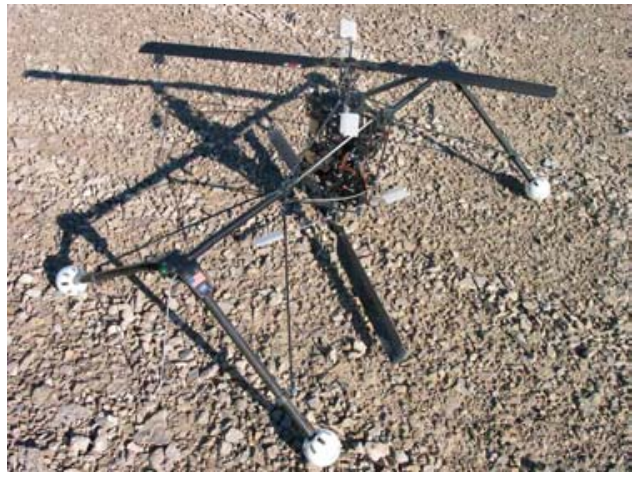

Figure 6. Smart Rotorcraft Field Assistants

As a consequence of this early pioneering work into vertical lift planetary aerial vehicles, the Division diversified its research interests into aerial explorers in general (fixed-wing as well as VTOL) and into bio-inspired autonomy. (Refer to, for example, the mission simulation illustration of a generic vehicle in Fig. 7; which can be thought of as a hybrid of the Refs. 21-22 vehicles). One of the key challenges for promoting aerial explorer missions is to demonstrate how the scientific return on investment can be maximized. Inherently, fixed-wing aerial explorers will have limited endurance and will likely only be able to sustain one flight per vehicle per mission, as these fixed-wing flyers will not be able to easily land and take-off again. Maximizing this return on investment can be accomplished by shifting the paradigm of aerial explorers from simple imaging and remote-sensing applications to one of a utility/carrier platform that can perhaps be best thought of as the ultimate manifestation of EDLS (Entry, Descent, and Landing System) technology ${ }^{20}$. Embracing this mission architecture concept, the aerial explorer becomes one (critical) element of a system of systems of sensors and robotic devices (air-deployed and otherwise), in effect comprising a small but potent robotic ecology of heterogeneous robotic systems (both internal and external autonomous agents). By embracing this paradigm shift, the flight duration of the aerial vehicle is of secondary importance as compared to the ability of the vehicle to accurately identify terrain features of interest and precisely, and safely, deploy sensors/devices. These air-deployed sensors and devices can then subsequently make high-value 
science measurements at the interesting surface locations, and to make sure that the deployed sensors/devices are imbued with a "persistence" well beyond the flight of the aerial explorer ${ }^{19}$.

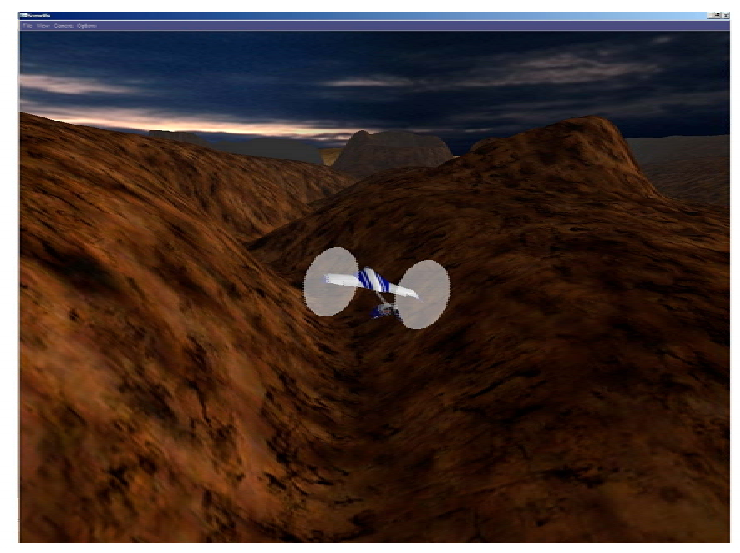

Figure 7. Aerial Explorer (Fixed-Wing) Mission Architectures

Aerial explorer mission architectures offer even more intriguing possibilities when considering the impact of using such robotic aerial assets in conjunction with human exploration. Expeditionary campaigns would derive considerably more mission flexibility by incorporating such "astronaut agents." Finally, many of the bio-inspired autonomy concepts and tools developed for aerial explorers can be potentially applied to other robotic/automated exploration systems. This is because many of the mission development challenges are common to all application domains wherein highly autonomous systems/robots must interact and efficiently work together - including humans - to accomplish the mission. (Such interaction between autonomous systems can be cooperating, neutral, or competing and either subordinate, coequal, or superior to each other).

\section{B. Autonomy and Design}

Recent studies into "bio-inspired autonomy" architectures ${ }^{19,20,23}$ have not only demonstrated the potential of this approach for autonomous control of aerial vehicles, but have suggested a natural migration path of this technology into a total system approach for an integrated autonomy and design suite of tools. Such an integrated autonomy and design approach would couple various design and software tools (at differing levels of modeling fidelity) into a suite of tools that mission planners can holistically use to define (and test through simulation) complete missions.

The bio-inspired autonomy architecture work being conducted at NASA Ames makes this a particularly tractable problem. This work would particularly be suited to address planetary/space missions that incorporate multiple autonomous systems and/or robots, i.e. a robotic ecosystem. This conceptualization of a mission being effected by a robotic ecosystem takes into account robotic/automated assets such as planetary aerial explorers, their cadre of robotic symbiotes, as well as the standard array of assets such as rovers, landers, and orbiters. The key outcome of this research would directly address the issue of autonomy as a critical design driver for vehicles and missions. Further, this should also address autonomy and design comprehensively at multiple levels. At the highest level, it would answer fundamental questions such as is it better for the prescribed mission objectives/constraints to have one sophisticated aerial explorer or multiple, simpler explorers? Is it better to employ a higher altitude, less maneuverable, longer-endurance aerial explorer for the mission versus a lower-altitude, more maneuverable, but shorter-range/endurance vehicle? What is the right mix of robots (pre-existing and deployed) for a given mission? At lower levels of mission specification, it would answer questions such as what is the right mix of robotic behaviors and initial prescribed scripting required for a given robot, for the given mission? What is the initial prescribed robot "personality" weight factors at the start of the mission? What are the optimal "anxieties" and associated constants used in the emotional modeling to drive the selection/implementation of robotic behaviors? Figure 8 illustrates some of the high-level elements of this notional integration of autonomy and design tools. 


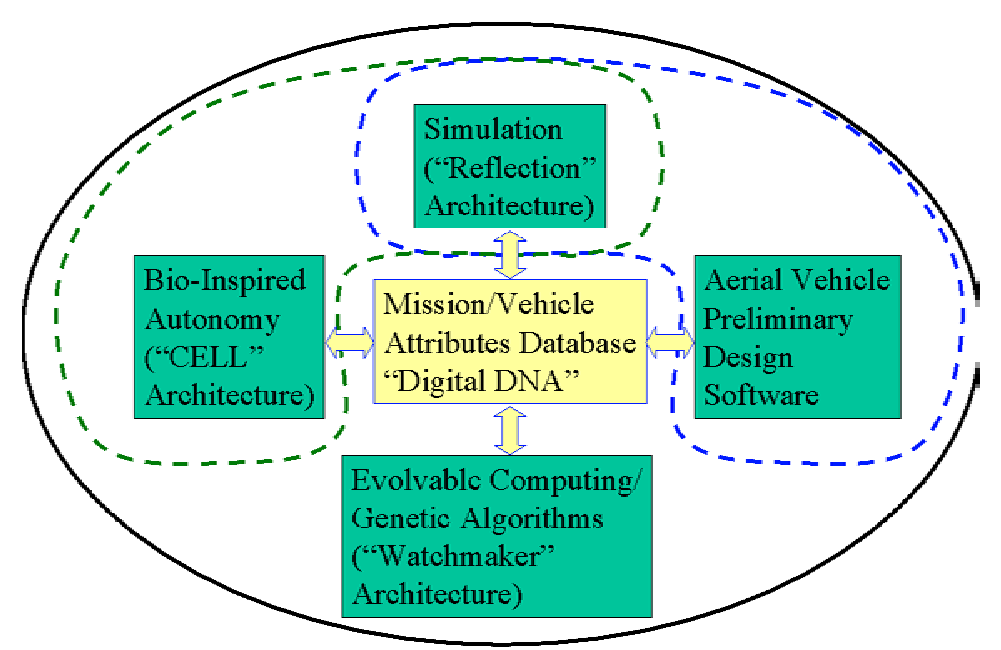

Figure 8. Integration of Various Software Tools

\section{Space Monkeys}

Research into aerial explorer autonomy and robotic symbiotes - as well as complementary work in bioinspired and biomimetic aerial systems ${ }^{1-2}$-- has led to a natural expansion of interest into other highly mobile types of robots. In this regard, there are many analogous aspects of aerial robots and in-space robots (used for inspection and maintenance of external spacecraft systems) - particularly the hybrid types of in-space robots that imbue both cold-gas thruster and biomimetic actuator control for mobility and mechanical manipulation.

Directly related to the safe and efficient operation of the CEV is the need for exterior inspections and the capability to perform low-level maintenance of the spacecraft. It is highly unlikely that EVA's of the CEV during deep-space transit will be feasible or desirable. Additionally, if the mission architecture calls for Mars orbital insertion by means of aerobraking, it will be necessary to inspect the spacecraft for damage. In both cases there is a clear case for a reliable semi-autonomous robotics -i.e. in-space micro-agents - for inspection and maintenance of the CEV and lander exterior surfaces and associated hardware. In-space micro-agents would complement external assets such as robotic service arms and Robonaut-type agents. Initial work in this area includes the "Personal Satellite Assistant ${ }^{24}$ (PSA)" and the JSC AERCam.

What is required is a semi-autonomous space micro-agent that is one part bio-inspired "space monkey" while the other draws inspiration from terrestrial autonomous aerial vehicles (Fig. 9). The envisioned device would have hybrid locomotion and effector capability - it would have cold-gas thrusters, but it would also have three robotic effectors for locomotion and manipulation. This would include an aft "grasping tail" and two forward, heterogeneous robotic arms. Locomotion, station keeping, and leverage would be provided by three different means: the robot would hold position or apply leverage by grasping exterior attach points with its "grasping tail"; it would be able to shift its position through movement of its "tail"; it would be able to catapult itself along the surface of the CEV by whip-like (but safe) motions of its tail; and it could use its cold-gas thrusters for free-flight for longer distances, or to make trajectory corrections after catapulting itself. 


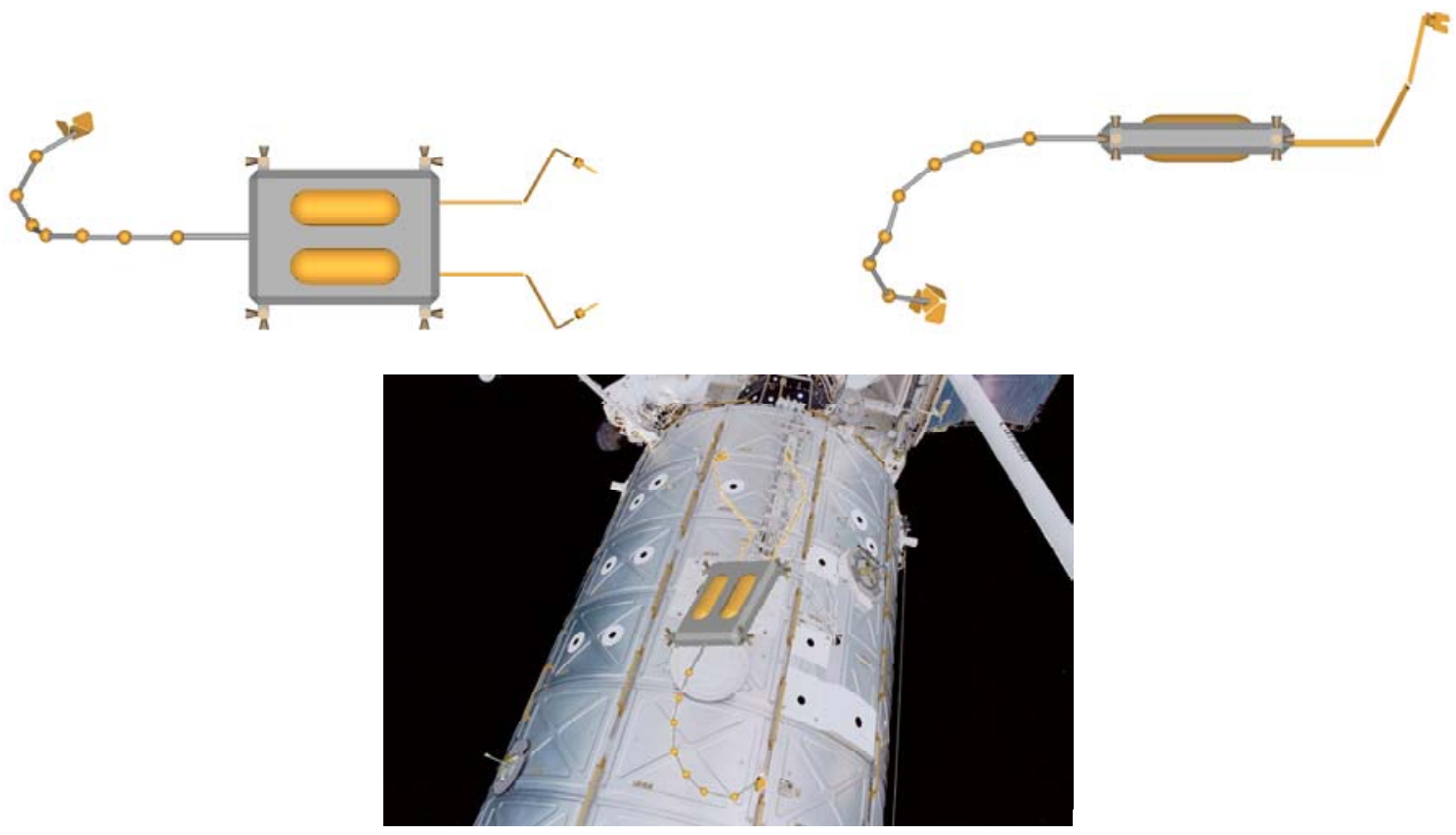

Figure 9. In-Space Hybrid Robot - with biomimetic "grasping tail" (Background image fromSTS100 of ISS exterior)

\section{Universal Anthropomorphic Processors}

The Division interests in system (classic mission planner and bio-inspired) autonomy, as well as a strong historical interest in man-in-machine human cognitive and behavioral modeling (such as the MIDAS software architecture), has led to development of the "Test Drive" and universal anthropomorphic processor (UAP) concepts. The UAP "black box" systems can be installed and used to teleoperate or semi-autonomously operate nominally human-rated exploration equipment for early risk mitigation (i.e. take the equipment for a "Test Drive") in relevant in-space or planetary surface environments. The intent is to make the UAP/Test Drive system of systems risk mitigation approach prevalent throughout the Project Constellation spiral development effort.

A key part of the "Test Drive" concept is the development of a "Universal Anthropomorphic Processor (UAP), a swap-out-able "black-box" computer/control unit that could substitute for a human operator during early developmental trials of field hardware. In addition to a human-operated mode, it could also enable equipment to have a teleoperated or even a semi-autonomous mode. The development of the UAP and embracing the "Test Drive" concept would enable a class of exploration vehicles that could be initially tested as robotic platforms as precursors to being used as human-rated transportation devices.

The UAP would be composed of both hardware and software components; the UAP software will be implemented in several "layers." Among these UAP software layers (in ascending anthropomorphic modeling complexity) would be a direct actuator control layer (directly connects to the teleoperation mode interface), a sensor/information input layer, a haptic/kinesthetic emulator layer, and a "human operator model" layer (Fig. 10). The UAP interface would be based in part in heritage human factors man and machine interface concepts developed at NASA ARC. The UAP is ultimately intended to be a portable device/system such that it could be easily interfaced with a large number of human-rated/control systems, given an appropriate specialized control/plant model for the system being operated by the UAP.

The "Test Drive" concept is a portfolio of robotic technologies (based primarily on the development of the UAP) supporting a common theme: test/demo something as a robot (Earth and Moon), perform some good science, and then (as per design) easily convert it into a human-rated capability/device. One of several examples of possible applications is to take a nominally human-rated lunar rover, make it a robotic platform, perform precursor mission(s) on the moon, and then show how to easily reconvert it back to being a human-rated transportation device and use for HRL phase of the exploration vision (Fig. 11).

Project Constellation has two key precepts underlying its overall conceptualization. First, robotic assets will both pave the way and act in partnership with human exploration. Second, the Moon is intended to be a proving 
ground for hardware and mission architectures for exploration to Mars and beyond. It should be possible to develop an integrated approach wherein robots - besides performing anticipated precursor science and survey work -- could actually effect human-rated equipment and vehicle "shakedown" trials on the Moon prior to human field operation. This is the underlying rationale of the "Test Drive" concept.

The "Test Drive" and UAP concepts could have a profound influence on CEV, HRL, and humanexploration of Mars by: 1. Lead to up-front design standards that require all equipment to not only be fly-by-wire or fly-by-light, but would also have easy interfaces near the human-rated-control locations to socket-in/access the onboard avionics by the UAP, 2. Would enable early test trials of human-rated hardware by testing them seamlessly as teleoperated/robotic devices, 3 . Would allow earlier scientific return on investment by the UAP enabling a greater host of robotic devices than perhaps otherwise possible (because of consolidating development paths between human-rated and robotic system development, and by standardizing the control architecture for both humanoperated and robotic systems). It is anticipated that there would be considerable engineering development costeffectiveness, and control interface risk-mitigation, through integration and usage of UAP into major Project Constellation systems that ultimately intended to be human-rated.

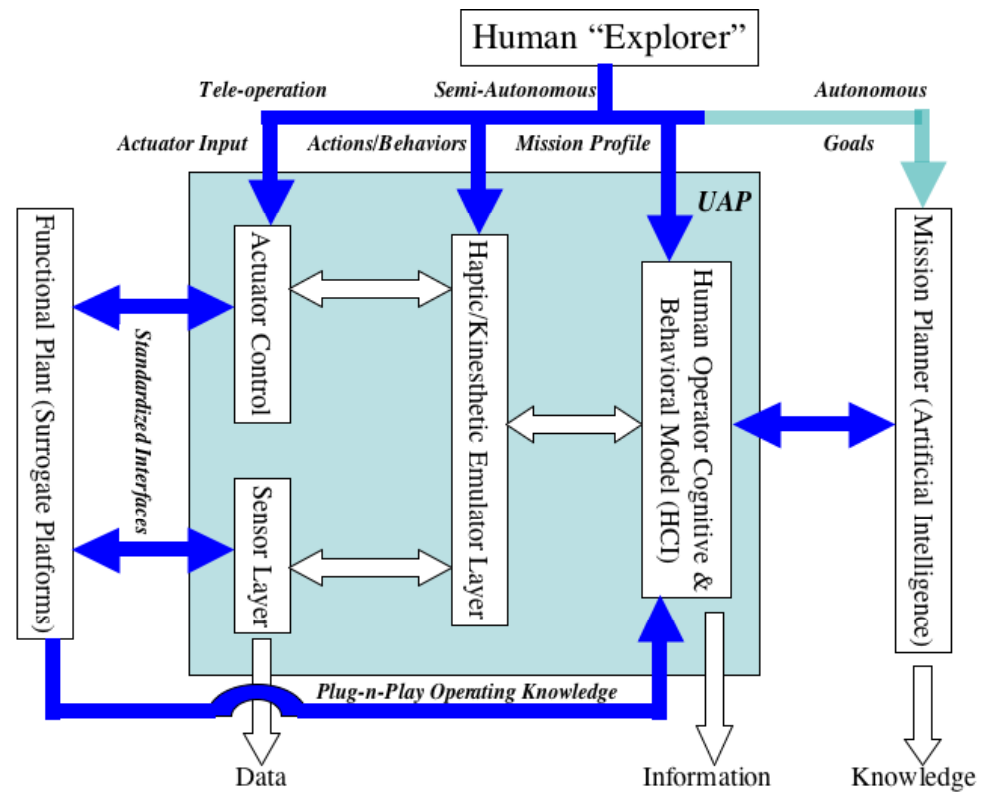

Figure 10. UAP Architecture
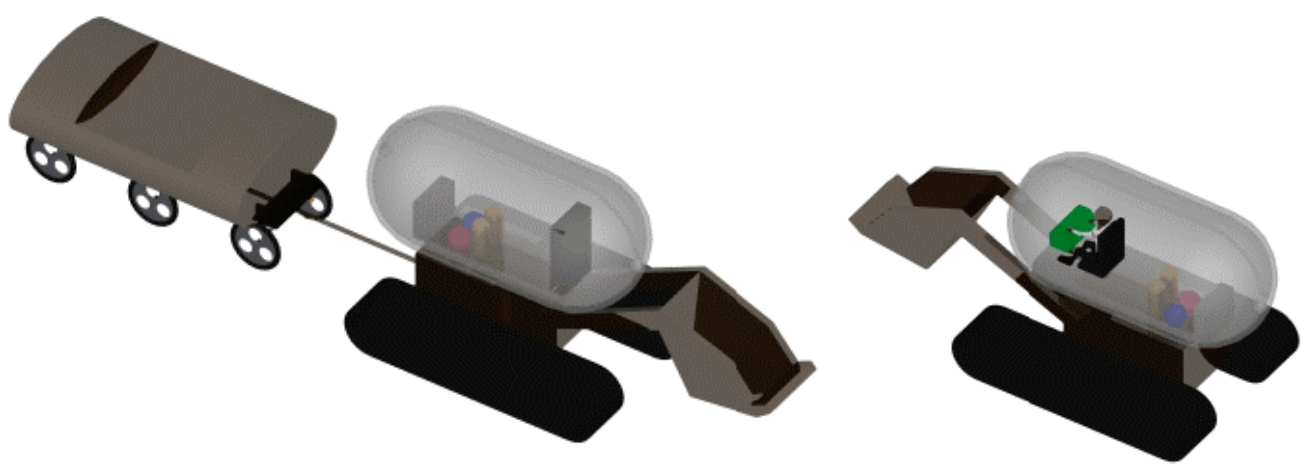

Figure 11. Test Drive/UAP-Enabled Equipment 


\section{E. BADDEST}

The Flight Vehicle Research and Technology Division has also advanced the concept of robotic lunar rovers that can fire ballistae to augment their overall "mobility" as well as perform specialized science functions otherwise unachievable (Fig. 12). This is the underlying premise of the BADDEST (Ballistic Assisted Delivery of Devices for the Efficient Study of Terrain) mission concept. The use of ballistic-launched sensor packages and robotic devices is an ideal mobility and science-instrument dissemination solution - given the low gravity and lack of an atmosphere -- for a robust robotic exploration campaign of the moon. Ballistae could encompass a wide range of sensors and other devices. Among these potential sensors/devices are: simple imagers, ground penetrators, and simple robots with limited localized mobility and/or effector capability. The potential of instrumented penetrators for near-surface lunar exploration is well recognized, but the complexity of deploying them from orbit at an airless body has made their use problematic. Deployment of penetrators from a lander would be straightforward and, of course, landing on the Moon has been accomplished many times. Ballistic probes could be launched to, or fly over, inaccessible or hazardous areas - including "cold traps." Additionally, some of the ballistic probes might be recoverable and repeatedly employed as "pop-up" scouts for terrain in advance of a planned rover trek. The BADDEST approach would be responsive to near-term lunar robotic explorations, support human lunar return, as well as make a complementary (shorter-range) adjunct to the use of aerial explorers for future Mars exploration.

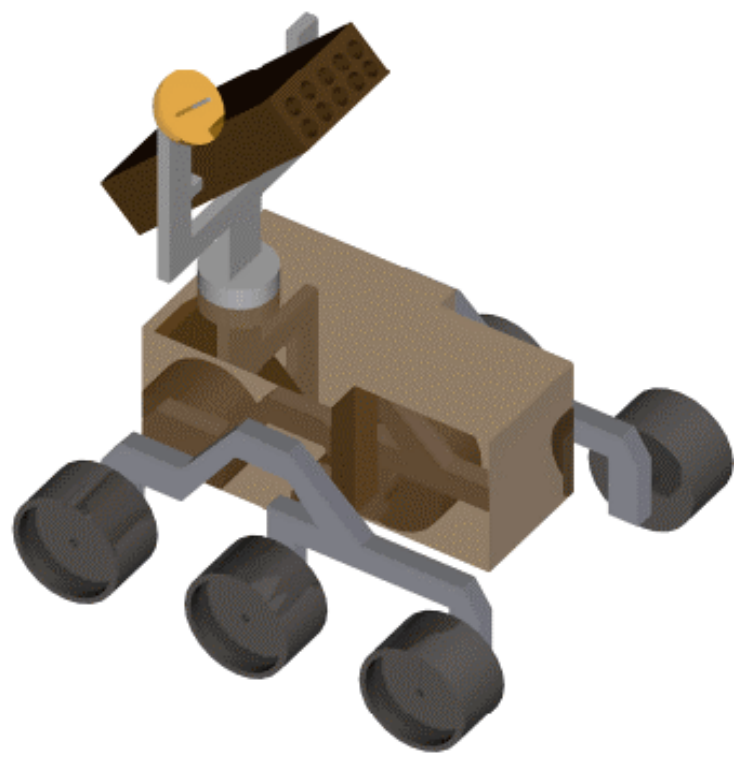

Figure 12. Notional Illustration of the BADDEST system (rocket-launcher version) on generic rover platform

\section{G. Rotary-Wing Aerobraking}

Many of the pre-cursor robotic missions to prepare for the human exploration of Mars could greatly benefit from advances in aeroassist and power-generation technologies. "Fixed" solar arrays to aid in (aeroassist) aerobraking orbital transfer/insertion has been previously employed on Magellan and Mars Global Surveyor missions. This aerobraking approach, though creative and efficient in application, has its limitations.

An innovative variation of the heritage fixed-array aerobraking approach has been advanced by the Flight Vehicle Research and Technology Division. Two sets of solar arrays would be implemented on the orbiter/spacecraft and both sets would be allowed to counter-rotate with respect to each other (reminiscent of a coaxial helicopter) as the spacecraft passes through the extreme edges of the planetary atmosphere (Fig. 13). These "rotors" could be used for aerobraking for orbital insertion of spacecraft, instead of bluff-body rigid surfaces (i.e., flat-panel solar arrays, ala Mars Global Surveyor) or inflatables, as has been demonstrated by the Russians in Earth orbit. The arrays would have pitch angle control such that not only would the arrays decelerate the spacecraft while 
passing through molecular traces of the upper atmosphere, but would also consequently transform some of this forward kinetic energy into angular momentum of the arrays. ("Pitch angle" being the array effective surface angle with respect to the spacecraft axis and forward motion.) The array hubs would be geared such that the arrays rotate in synchronized opposite directions, and so very little residual torque would be imparted to the spacecraft. Not only would the array hub/shafts have intermesh gearing, but this gear-box would also be coupled to an synchronous rotating electric generator. The advantage of "rotors" for aerobraking is enhanced spacecraft trim/control (given array pitch-angle control mechanisms) during orbital insertion. Secondly, and here is the primary advantage, the rotors' utility in generating electrical power (gaining a twice-fold return on bleeding off the spacecraft kinetic energy) during the perigee portion of the orbital passes. Particularly power intensive tasks could be performed using stored electrical power, from ultra-capacitors/batteries, from the aerobraking maneuvers. This power could be used for high-power, long-range sensors/instrumentation and/or used to power spacecraft ion-thrusters for additional orbital braking and steering control.

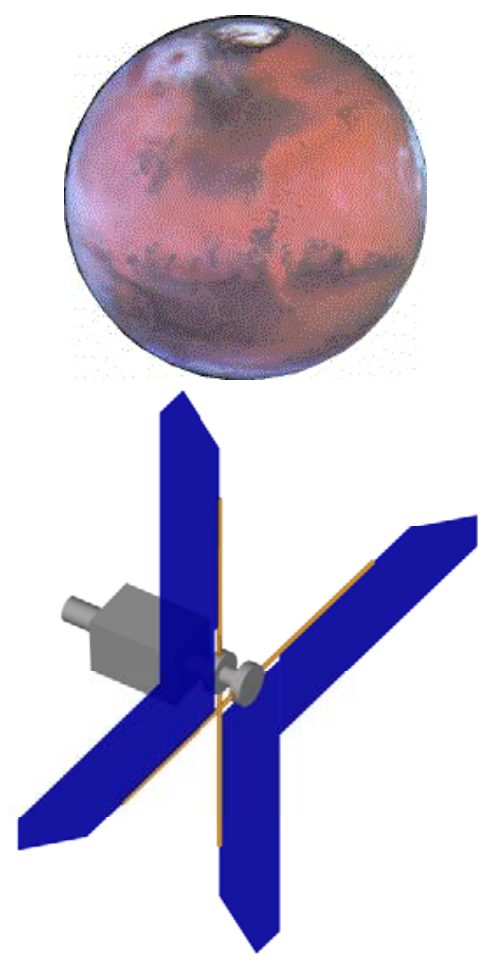

Figure 13. "Rotary-Wing" Aerobraking

\section{H. Rotary-Wing Decelerators and Reentry Vehicles}

There is a critical need for CEV reentry vehicle designs that maximize safety and flexibility, while dealing with higher entry velocities than seen with LEO missions. Apollo-type landings in the ocean will potentially be viewed as primitive and unsafe. Control of the spacecraft's descent through the atmosphere and of its landing calls for new technology developments where vertical lift research community can offer essential skills (aerodynamics, advanced vehicle design, system analysis, and, notably, rotary-wing technology) and innovation.

In the past, serious consideration was given to developing rotary-wing reentry vehicles (wherein autorotating rotors provided partial or full aerodynamic deceleration instead of parachutes). Rotary-wing reentry vehicle work (as applied to capsule-type vehicles) was performed at Kaman ${ }^{10}$ (circa 1961) and Ames ${ }^{11}$ (circa 1968). In the case of the Kaman and Ames work, the aerodynamic characteristics of autorotating rotors were measured from supersonic (Mach 2-3) to subsonic speeds. Recently the rotary-wing reentry vehicle concept has been revisited. In addition to application to capsule-type entry vehicles, Ames researchers have also examined, to a limited degree, application of rotary-wing reentry vehicle technology to slender-body space planes (Fig. 14). 


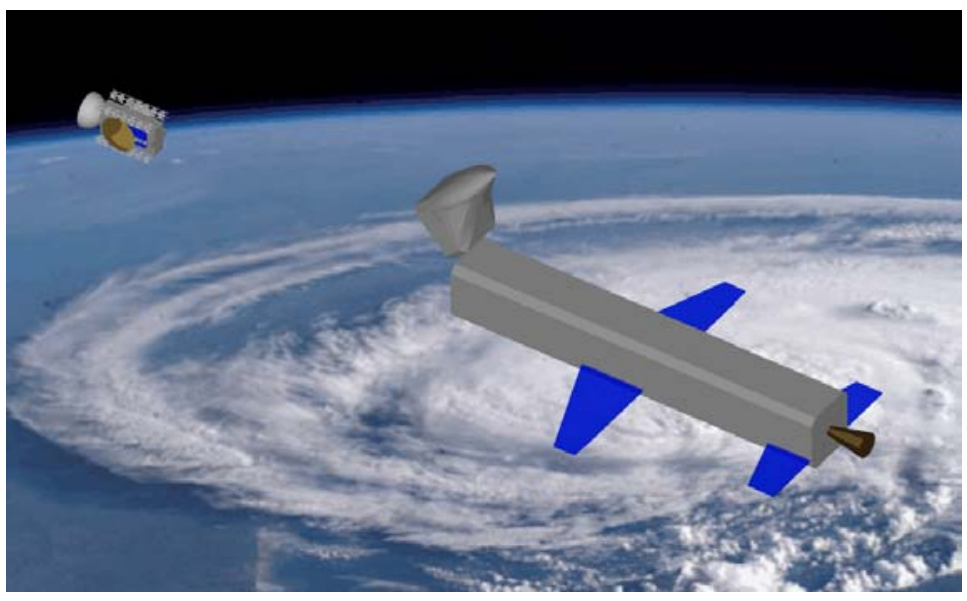

Figure 14. Slender-body Space Plane Automated Satellite Launch for LEO

The potential merits of rotary-wing reentry vehicles are many. First, improved L/D with deployment of rotor(s) at 10-50k $\mathrm{ft}$ altitude. Second, improved maneuverability and handling qualities during final landing stage (5-10k ft altitude) for increased safety at the landing site. Third, autorotating rotors for reentry vehicles provide for the ability to do "soft flare landing." In particular, a slender-body space plane incorporating autorotating rotors has unique advantages over a capsule-type configuration, with and without rotors. These unique advantages include: 1 . high levels of L/D throughout all flight regimes, i.e. wings and rotors can be optimized for hypersonic/supersonic and subsonic regimes respectively; 2. take-off and landing aborts can be handled with increased safety with the reentry vehicle acting as a gliding autogyro (including potentially landing at rough, unprepared landing sites). Figure 15a-c illustrates the deployment of the rotary-wing decelerators from a slender-body space plane.
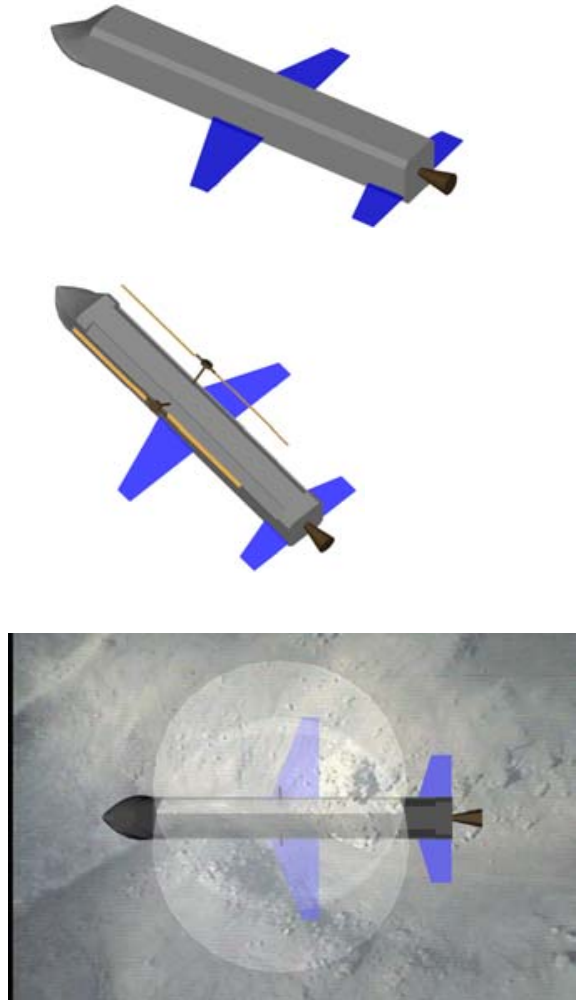

Figure 15. Rotary-Wing Reentry Vehicle: (a) early-, (b) mid-deployment, \& (c) autorotation/soft-flare landing

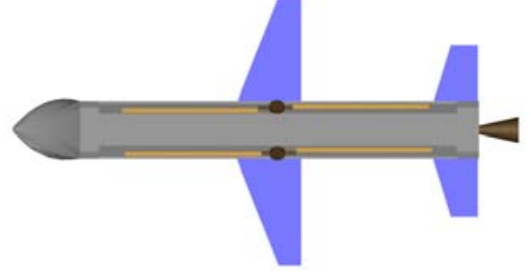

(a)

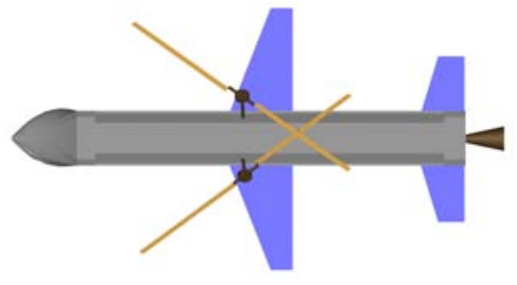

(b)

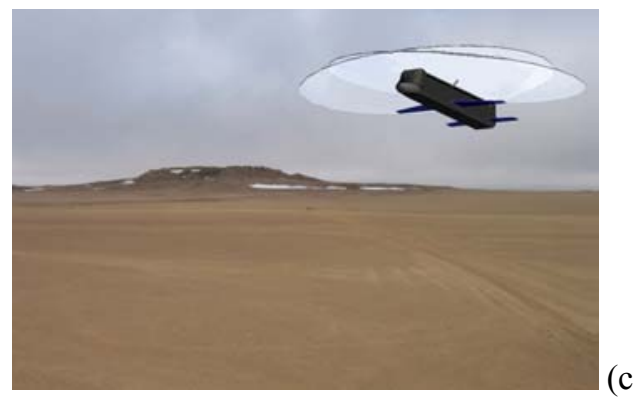

(c) 


\section{Human-Rated Landers and VTOL Experience}

While much can be inherited from advanced robotic soft landers, it seems safe to say that a human-rated lunar or martian lander would be much more complex and physically much larger than any robotic lander (thus changing the nature of surface hazards). The development of autonomous terminal hazard avoidance for robotic Mars landings is a major challenge because of the complexity of the sensing and control capability needed. Terminal hazard avoidance is something for which human pilots have exceptional sensing and control capabilities and, thus, any human-rated planetary lander will certainly be designed for human override and control; such override and control technology is more aligned with VTOL aircraft control than with robotic systems. Irrespective of the ultimate destination - Mars or the Moon - or the favored mission architecture for Project Constellation, one thing seems to be assured and likely to be relatively invariant with respect to the technologies required: human-rated lander configurations will need to be both resurrected and/or developed. A facility for development, test, and training akin to that required for Apollo (such as the LLRV and LLRF) will be needed. Additionally precursor surrogate vehicle testing will also be in order (Fig. 16).

At least three types of crewed lunar landers have been contemplated in past lunar reference missions: an excursion lander that supports a crew of two for missions for short duration excursions, "habitat" landers that could support long duration missions lasting approximately 30 days or greater, and autonomous "cargo" landers for emplacement of advance supplies and equipment.

Landers, for extended lunar or Mars missions, will have to function as "home away from home," to some degree - more so than the Apollo missions LEMs. This will entail more forethought as to the design of these future generation landers. Correspondingly, the landers and/or surface habitats will also have to support different classes of robotic lunar explorers to augment the crewed lander excursions/missions. These lunar robotic explorers could be of several types and their development and introduction into the lunar architecture could be performed in stages. Among the possible robotic lunar explorers that could be developed are teleoperated and semi-autonomous probes, rovers, and landers/hoppers (operated from the lunar or planetary surface, or the ISS and Earth, if the communication time delay can be tolerated). Operation of such robotic lunar explorers might entail the incorporation of a science officer station in the landers and/or surface habitats (a "virtual world' environment) for robotic exploration operation and data gathering/analysis.

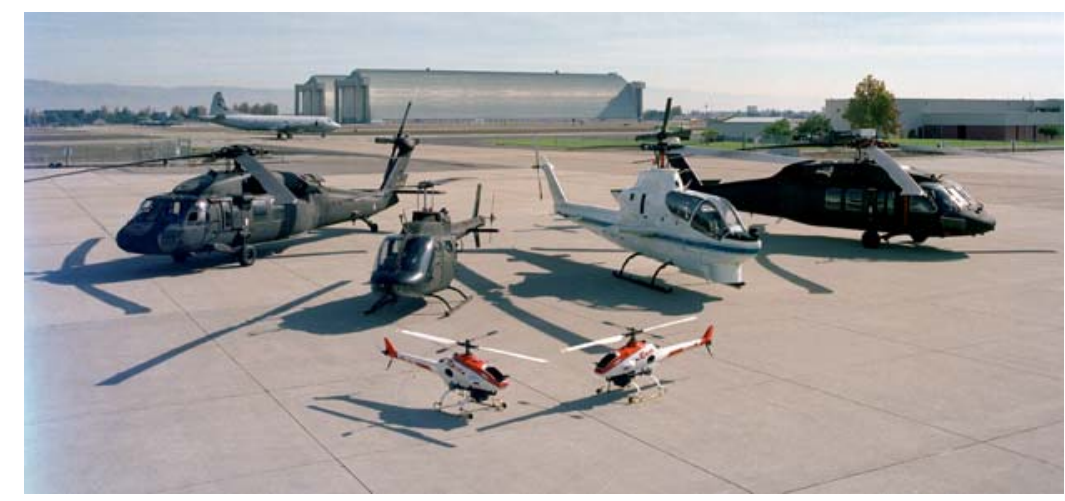

Figure 16. Lander Technology Development: initially can use surrogate VTOL vehicles

There are many technology analogs between intelligent/autonomous flight (take-off \& landing) of VTOL aircraft and robotic/crewed lunar landers. The Flight Vehicle Research and Technology Division and the Intelligent Systems project are currently supporting Autonomous Rotorcraft research that has dual-application potential to lunar and Mars lander development ${ }^{14-15}$. Additionally, work in the Intelligent Vehicle Health Management (IVHM) area at Ames also has important implications for the development of the human-rated landers. Finally, and perhaps most importantly, human-factors and control law expertise (including the use of fixed- and motion-based simulators) will be essential for the conceptualization and development of human-rated landers for lunar and/or Mars exploration. 


\section{Concluding Remarks}

A balanced portfolio of aeronautic and astronautic technologies in this new era of exploration is essential. Given both past history and recent experience within the Ames Research Center's Flight Research and Technology Division such balance can be achieved. In fact, there is a largely unexplored mother lode of leveraged/dual-use technologies and concepts from the aeronautics and vertical lift research communities that can be applied to help make the exploration vision a reality. This paper briefly summarized some of these technologies and concepts.

\section{Acknowledgments}

The innumerable and invaluable technical and programmatic contributions of Geoffrey Briggs (Science Director, NASA Ames Center for Mars Exploration), Matt Whalley (U.S. Army Aeroflightdynamics Directorate), Greg Pisanich (QSS Group, Computational Sciences Division), and Corey Ippolito (QSS Group, Computational Sciences Division) to the work summarized in this paper are gratefully acknowledged. Finally, this is for Courtney, my own future explorer.

\section{References}

${ }^{1}$ Aiken, E.W., Ormiston, R.A., and Young, L.A., "Future Directions in Rotorcraft Technology at Ames Research Center," $56^{\text {th }}$ Annual Forum of the American Helicopter Society, International, Virginia Beach, VA, May $2-4,2000$.

${ }^{2}$ Young, L.A., Aiken, E.W., Johnson, J.L., Demblewski, R., Andrews, J., and Klem, J., "New Concepts and Perspectives on Micro-Rotorcraft and Small Autonomous Rotary-Wing Vehicles," AIAA $20^{\text {th }}$ Applied Aerodynamics Conference, St Louis, MO, June 24-27, 2002.

${ }^{3}$ Plice, L., Pisanich, G., Lau, B., and Young, L.A., "Biologically Inspired 'Behavioral' Strategies for Autonomous Aerial Explorers on Mars," IEEE Aerospace Conference, Big Sky, MT, March 2003.

${ }^{4}$ Plice, L., "Robot Economy," Robosphere 2002: Workshop on Self-Sustaining Robot Ecologies, NASA Ames Research Center, Moffett Field, CA, November 2002.

${ }^{5}$ Pisanich, G. and Young, L.A., "An Aerobot Ecology," Robosphere 2002: Workshop on Self-Sustaining Robot Ecologies, NASA Ames Research Center, Moffett Field, CA, November 2002.

${ }^{6}$ Young, L.A., Aiken, E.W., and Briggs, G.A., "Smart Rotorcraft Field Assistants for Terrestrial and Planetary Science," 2004 IEEE Aerospace Conference, Big Sky, MT, March 2004.

${ }^{7}$ Pisanich, G., Young, L.A., Ippolito, C., Plice. L., Lau, B., and Lee, P., "Initial Efforts towards MissionRepresentative Imaging Surveys from Aerial Explorers," SPIE (International Society of Optical Engineers) Electronic Imaging Conference, San Jose, CA, January 2004.

${ }^{8}$ Pisanich, G., Ippolito, C., Plice, L, Young, L., and Lau, B., "Actions, Observations, and Decision-Making: Biologically Inspired Strategies for Autonomous Aerial Vehicles," AIAA Aerospace Sciences Conference, Reno, NV, January 2004.

${ }^{9}$ Young, L.A., et al, "Rotary-Wing Decelerators for Probe Descent through the Atmosphere of Venus," $2^{\text {nd }}$ International Workshop of Planetary Probes, NASA Ames Research Center, August 2004.

${ }^{10}$ Ham, N., "An Experimental and Theoretical Investigation of a Supersonic Rotating Decelerator," Journal of the American Helicopter Society, Vol. 8, No. 1, January 1963.

${ }^{11}$ Levin, A.D and Smith, R.C., "Experimental Aerodynamics of a Rotor Entry Vehicle," AIAA Aerodynamic Deceleration Systems Conference, El Centro, CA, September 23-25, 1968.

${ }^{12}$ Hudson, G., "The ROTON Concept and It's Unique Operations," Proceedings of 6th ISCOPS, AAS Vol. 91, AAS, pp. 529-541.

${ }^{13}$ Stepniewski, W.Z. and Shinn, R.A., "Soviet vs. U.S. Helicopter Weight-Prediction Methods," $39^{\text {th }}$ Annual Forum of the American Helicopter Society, St. Louis, MO, May 9-11, 1983.

${ }^{14}$ Whalley, M., et al, "The NASA/Army Autonomous Rotorcraft Project," 59th Annual Forum of the American Helicopter Society, Phoenix, AZ, May 2003. 
${ }^{15}$ Whalley, M., et al, "Design, Integration, and Flight Test Results for an Autonomous Surveillance Helicopter," AHS International Specialists' Meeting on Unmanned Rotorcraft: Design, Control and Testing, Chandler, AZ, January 18-20, 2005.

${ }^{16}$ Datta, A, Roget, B., Griffiths, D., Pugliese, G., Sitaraman, J., Bao, J., Liu, L., and Gamard, O., "Design of the Martian Autonomous Rotary-Wing Vehicle," AHS Specialist Meeting on Aerodynamics, Acoustics, and Test and Evaluation, San Francisco, CA, January 2002.

${ }^{17}$ Thompson, B., "Full Throttle to Mars," Rotor \& Wing, Phillips Business Information, LLC, Potomac, MD, March 2001.

${ }^{18}$ Wu, C.C., Ofoma, U., and He, C., "Autonomous UAV Concept for Titanic Missions," $60^{\text {th }}$ Annual Forum of the AHS, International, Baltimore, MD, June 7-10, 2004.

${ }^{19}$ Young, L.A., Ippolito, C., and Pisanich, G., “Aerial Explorers," 43 ${ }^{\text {rd }}$ AIAA Aerospace Sciences Meeting, Reno, NV, January 2005.

${ }^{20}$ Young, L.A. and Pisanich, G., "Aerial Explorers and Robotic Ecologies," Second International Conference on Computing, Communication and Control Technologies, Austin, TX, August 14-17, 2004.

${ }^{21}$ Guynn, M.D., et al, "Evolution of a Mars Airplane Concept for the ARES Mars Scout Mission," $2^{\text {nd }}$ AIAA "Unmanned Unlimited" Systems, Technologies, and Operations, AIAA 2003-6578, San Diego, CA, September 15-18, 2003.

${ }^{22}$ Malin, M., et al, "Mars Airborne Geophysical Explorer (MAGE) Discovery Mission Proposal," http://www.msss.com/mage release/.

${ }^{23}$ Ippolito, C., Pisanich, G., and Young, L.A., "Cognitive Emotion Layer Architecture for Intelligent UAV Planning Behavior and Control,” 2005 IEEE Aerospace Conference, Big Sky, MT, March 2005.

${ }^{24}$ Dorais, G.A. and Gawdiak, Y., "The Personal Satellite Assistant: an internal spacecraft mobile monitor," 2003 IEEE Aerospace Conference, Big Sky, MT, March 2003. 